\title{
Effects of atmospheric refraction and turbulence on long-range IR imaging in the marine surface layer Comparisons between experiment and simulation
}

\author{
G. J. Kunz ${ }^{* a}$, A.M.J. van Eijk ${ }^{\mathrm{a}}$, D. Tsintikidis ${ }^{\mathrm{b}}$ and S.M. Hammel ${ }^{\mathrm{b}}$ \\ ${ }^{a}$ TNO Defence, Security and Safety, The Hague, The Netherlands, \\ ${ }^{\mathrm{b}}$ SPAWAR Systems Center, San Diego, USA
}

\begin{abstract}
EOSTAR, a PC based Windows application, integrates the required modules necessary to calculate the electro-optical sensor performance on the basis of standard meteorological data. The primary output of EOSTAR consists of the synthetic sensor image ("what does the sensor see?") and a coverage diagram ("detection probability versus range"). As part of the EOSTAR validation effort, the refraction and turbulence modules are being evaluated against literature data, similar models and experimental results. It is shown that the EOSTAR model can predict with reasonable success the occurrence of optical turbulence and refraction phenomena such as mirages. The major cause for discrepancies between the various models is attributed to the underlying micrometeorological bulk modules, whereas the sensitivity of the predictions on the values of the meteorological input parameters is held responsible for the discrepancies between model predictions and measurements.
\end{abstract}

Keywords: atmospheric refraction, turbulence, image distortion, scintillation

\section{INTRODUCTION}

Infrared (IR) sensors are attractive in a multitude of operational conditions, especially during RF silence or for the detection of small targets at lower altitude in the marine environment. However, the performance of IR systems depends strongly on the atmospheric conditions, and degrading may occur due to transmission losses, refraction and turbulence. The resulting image distortion may not only hamper detection, but also complicate the classification and identification process. For a proper assessment of platform vulnerability ("can I detect the threat?") and the probability of mission accomplishment, it is vital to assess the performance of sensor systems under the prevailing environmental conditions.

Models are available to predict the performance of IR sensors in the marine atmospheric surface layer on the basis of standard meteorological observations. Their primary use is in mission planning tools, where they provide the maximum range at which a surveillance or threat sensor can detect a target. Alternatively, these tools serve educational purposes by providing insight in the atmospheric phenomena. EOSTAR, a PC based Windows application, integrates the required models necessary to calculate the sensor performance on the basis of standard meteorological data. The primary output of EOSTAR consists of the synthetic sensor image ("what does the sensor see?") and a coverage diagram ("detection probability versus range").

The EOSTAR development is a continuing, co-operative effort between TNO Defence, Security and Safety (The Netherlands) and SPAWAR Systems Center (USA). Several papers report on the initial development and validation efforts of the model ${ }^{1,2}$ (see also references therein). In this contribution, we focus on the performance of EOSTAR with respect to predicting optical turbulence and refraction phenomena such as mirages. To this end, a survey has been made of the literature, where a wealth of information can be found on observations of refraction phenomena and the performance of models to predict these. EOSTAR is compared to those observations and other models. Furthermore,

\footnotetext{
* Contact information: Gerard.Kunz@tno.nl, phone: +31 70374 0460, TNO Physics and Electronics Laboratory, P.O. Box 96864, 2509 JG The Hague, The Netherlands; http://www.tno.nl
}

Atmospheric Optical Modeling, Measurement, and Simulation, edited by Stephen M. Doss-Hammel, Anton Kohnle, Proc. of SPIE Vol. 5891, 58910A, (2005) · 0277-786X/05/\$15 · doi: 10.1117/12.614884 
experimental data of optical turbulence as recorded during the VAMPIRA trial is compared to EOSTAR model predictions.

\section{THE EOSTAR MODEL}

The EOSTAR (Electro-Optical Signal Transmission And Ranging ${ }^{1,2}$; www.tno.nl/eostar) model assesses electro-optical propagation phenomena on sensor performance in the maritime atmospheric surface layer. EOSTAR incorporates micrometeorological bulk models and molecular and aerosol transmission codes. Furthermore, the model utilizes a ray tracer to calculate the optical path between target and sensor. The target is represented by a collection of structural elements with specific properties such as reflectivity and heat capacity. A heat flux balance model ${ }^{3}$ evaluates the signature of the target under the selected environmental conditions. An empirical maritime background model allows for a sea/sky background with or without clouds. All these modules are integrated in a mouse-driven Windows application that allows the user to focus on individual phenomena (such as transmission) or to view the major (overall) products of the code.

The major products of EOSTAR are the synthetic sensor image, which consists of a view of the target against its background under the selected environmental conditions. The user may opt to view the overall effect of all propagation phenomena, or to view only the effect of, e.g., scintillation. The second major product of EOSTAR consists of coverage diagrams, which represent detection range of the chosen sensor against a specific threat. Here, the user may view 2Dcoverage diagrams in the $\mathrm{X}-\mathrm{Z}$ (height-range) domain or X-Y (azimuthal) domain.

As mentioned before, the validation of the EOSTAR model is currently underway. At present, single modules governing aerosol absorption, maritime backgrounds and target signatures have been validated individually. The performance of the bulk micrometeorological models has also been assessed. These models provide the elementary parameters for calculating the micrometeorological condition, the vertical profiles of refractivity, ray trajectories and turbulence effects, using standard or bulk meteorological observations such as wind speed, air- and surface temperature, humidity and wind speed. Due to the large number of non-linear equations involved, the bulk models are solved iteratively. Several choices are possible for the critical variable(s) controlling the loop, which has led to a family of different bulk models ${ }^{4-8}$. However, despite these differences there is a good agreement between the outputs of the models for unstable atmospheric conditions. Larger differences are found under stably stratified conditions due to the occurrence of singularities in the solutions. EOSTAR incorporates various bulk models and an inter comparison of these models has been made with test data available in literature. Although the various models generally agreed well, excellent results were obtained for the Smith approach ${ }^{4}$ and Paulson's ${ }^{9}$ stability functions.

In this contribution we focus on the performance of the EOSTAR modules for refraction and optical turbulence. The module to calculate refraction effects has extensively been discussed elsewhere. ${ }^{1}$ Although EOSTAR is a model for electro-optical wavelengths, recently a module was added that evaluates the refractive index of air at radio frequencies. ${ }^{10}$ This module allows us to calculate the evaporative duct height (EDH), which provides access to additional experimental data to validate the bulk models and ray tracer.

Optical turbulence is caused by variations in the refractive index of air due to spatial and temporal atmospheric variations induced by wind shear and/or convective processes of temperature and humidity. Therefore, optical and infrared turbulence are closely related to atmospheric turbulence. In accordance with other turbulent quantities, optical turbulence is expressed in the structure function $D_{n n}(R)$, which is defined as the ensemble average of the refractive index difference $<\left(n_{R 2}-n_{R I}\right)^{2}>$ between two points of observation separated by a distance $R=R_{2}-R_{1}$. Mathematically the structure function is written as:

$$
D_{n n}(R)=\left\langle\left(n_{R 2}-n_{R 1}\right)^{2}\right\rangle=\left\langle(d n)^{2}\right\rangle
$$

Dimensional analysis shows that the structure function is related to the refractive index structure parameter $C_{n}{ }^{2}$ and the distance between the points of observation. According to the notation of, e.g., Beland ${ }^{11}$ the relation between the structure function and the refractive index structure parameter is written as: 


$$
D_{n n}(R)=C_{n}^{2} R^{2 / 3}
$$

To relate the micrometeorological turbulence to optical turbulence the variation of the refractive index is written in terms of variations of air temperature, humidity and pressure:

$$
d n=\frac{\partial n}{\partial T} d T+\frac{\partial n}{\partial Q} d Q+\frac{\partial n}{\partial P} d P
$$

Because pressure variations in atmospheric eddies are small and have only a slight influence on the refractive index variations, the last term in equation (3) can be ignored. Substitution of the square of the refractive index variations in equation (1) results in:

$$
D_{n n}(R)=\left\langle\left(\frac{\partial n}{\partial T} d T\right)^{2}+\left(\frac{\partial n}{\partial Q} d Q\right)^{2}+2\left(\frac{\partial n}{\partial T} \frac{\partial n}{\partial Q} d T d Q\right)\right\rangle
$$

The last term in this equation describes the eddy covariance between temperature and humidity. Rewriting of equation (4) in terms of structure functions like equation (1) results in:

$$
D_{n n}(R)=\left(\frac{\partial n}{\partial T}\right)^{2} D_{T T}(R)+\left(\frac{\partial n}{\partial Q}\right)^{2} D_{Q Q}(R)+2\left(\frac{\partial n}{\partial T} \frac{\partial n}{\partial Q}\right) D_{T Q}(R)
$$

Substitution of the structure function parameters for the refractive index, temperature, humidity and the covariance between temperature and humidity results in:

$$
C_{n}^{2}=\left(\frac{\partial n}{\partial T}\right)^{2} C_{T}^{2}+\left(\frac{\partial n}{\partial Q}\right)^{2} C_{Q}^{2}+2\left(\frac{\partial n}{\partial T} \frac{\partial n}{\partial Q}\right) C_{T Q}
$$

This important equation describes the relation between atmospheric turbulence and optical/infrared turbulence. The dependence of the refractive index of air on temperature and humidity can be found elsewhere. ${ }^{12,13}$. The structure function parameters for temperature and humidity can either be measured or estimated from the scaling temperature, scaling humidity and stability using equations (7a-c):

$$
\begin{aligned}
& {C_{T}{ }^{2}}^{2} z^{-2 / 3} T_{*}^{2} f_{1}(z / L) \\
& C_{Q}{ }^{2}=z^{-2 / 3} Q_{*}{ }^{2} f_{2}(z / L) \\
& C_{T Q}{ }^{2}=z^{-2 / 3} T_{*} Q_{*} f_{3}(z / L)
\end{aligned}
$$

Expressions for the empirical functions $f_{1}, f_{2}$ and $f_{3}$ can be found in literature. ${ }^{14-22}$ Scintillation is caused by the randomly variable, normally distributed, real part of the propagation constant $\sigma_{\chi}$. The Rytov approximation predicts the following relation between $\sigma_{\chi}$ and the structure function parameter for the refractive index $C_{n}{ }^{2}$ over a non-homogeneous path $R:^{11,23-25}$

$$
\sigma_{\chi}^{2}(R)=0.56\left(\frac{2 \pi}{\lambda}\right)^{7 / 6} \int_{0}^{R} C_{n}^{2}(r)\left(\frac{r}{R}\right)^{5 / 6}(R-r)^{5 / 6} d r
$$

In the case of isotropic turbulence equation (8) reduces to: ${ }^{26,27}$ 


$$
\sigma_{\chi}^{2}=0.124 C_{n}^{2}\left(\frac{2 \pi}{\lambda}\right)^{7 / 6} R^{11 / 6}
$$

Equations (8) and (9) are only applicable for weak turbulence $\sigma_{\chi}^{2}<0.3\left(\sigma_{\chi}<0.55\right)$. The random, normally distributed, variations of the propagation constant leads to a log-normal distribution of the intensity variations. Using the property of this distribution, ${ }^{11,28}$ the variance of the normalized intensity fluctuations can be described by:

$$
\frac{\sigma_{I}^{2}}{\bar{I}^{2}}=e^{4 \sigma_{\chi}^{2}}-1
$$

This property is experimentally accessible: the variations in normalized transmission provide thus basic information on the refractive index structure function parameter $C_{n}{ }^{2}$. However, equation (10) is derived under the assumption of a point source and a point receiver. For receivers with a finite diameter the signal variations are suppressed due to aperture averaging and another equation ${ }^{26,27}$ should be used for calculating $C_{n}{ }^{2}$ time series from the variance of the lognormalized signal of a transmissometer:

$$
\sigma_{\ln I}^{2} \approx 0.9 C_{n}^{2} R^{3} D^{-1 / 3}
$$

where $D$ is the receiver diameter. This equation can be used under the condition that $D>>(\lambda R)^{0.5}$.

\section{MODEL PERFORMANCE - REFRACTION}

In this section, we discuss the EOSTAR performance for prediction of refraction effects. The evaluation has been made on the basis of literature data, to which we have applied EOSTAR. For the sake of conciseness, we will not fully describe the experiments underlying the data, and in some cases, we will refer to tables and figures of the original publications. The overall conclusions will be presented at the end of this section.

Dion et al. ${ }^{29}$ publish two examples of refraction effects in the visible and the 3-5 $\mu \mathrm{m}$ spectral band over a path length of $11.9 \mathrm{~km}$, including sufficient information to simulate the propagation paths. The ray trajectories and thus the ray directions at the location of the source calculated with their WKDMBL compare well with the values predicted (now) by EOSTAR, in both examples. The experimentally observed mirage images were also predicted by both models. EOSTAR also confirms Dion's conclusion that refraction effects in the visible and in the IR band are comparable. This conclusion may seem surprising at first sight, since the refractive index of air is a function of wavelength in the electro-optical domain. However, refraction effects such as mirages depend on the vertical gradient of the refractivity $(d N / d z)$, which is almost independent of wavelength.

Forand et al. $^{30}$ present three cases for the visible and the 3-5 $\mu \mathrm{m}$ bands measured during the MAPTIP experiment, all under unstable atmospheric conditions. Two cases concern the measured mirage effects of point sources mounted on a ship, which compare favorably with predictions of their WWKD model. Similar predictions are now found with EOSTAR. The third case concerns the measured angular elevation of a series of lamps mounted on a stable platform at a distance of $10 \mathrm{~km}$. Although the trend in the measured data can be predicted by EOSTAR, the absolute values of the observed elevations of the point sources show an offset of $0.5 \mathrm{mrad}$, which cannot satisfactorily be explained from uncertainties in meteorological data. Possible reasons for this discrepancy are an offset in angular elevation of the camera or the effect of tide on the height of the point sources above the water surface.

Dion $^{31}$ presents results obtained with the WKD model for trials in coastal waters. For a specific case, he calculates values of the horizon-limited range (HLR) under non-refractive and refractive conditions as $22.6 \mathrm{~km}$ and $17.6 \mathrm{~km}$ respectively. Using EOSTAR, we now predict $22.6 \mathrm{~km}$ and $16.7 \mathrm{~km}$, respectively. Figure 2 of Dion's paper then shows the evolution of the HLR for other meteorological conditions. Although the general trend in the data is reproduced by EOSTAR, we observe a systematic offset (cf. the numerical example given above) for which we have no explanation. EOSTAR does confirm Dion's ${ }^{29,31}$ observation that the shape of the refractivity profile is independent of wavelength. As mentioned in the previous section, EOSTAR now also contains a module to calculate the evaporative duct height. This quantity is also 
supplied by the WKD model, and a comparison with EOSTAR shows that EOSTAR predicts the trends correctly, but differs again in absolute values (10-50\% higher). A possible explanation may be the sensitivity of the duct height on the exact meteorological conditions.

Forand $\mathrm{d}^{32}$ publishes more results for the series of lights at various heights as used in the MAPTIP experiment. The vertical transfer functions ${ }^{1}$ obtained over a distance of $10.4 \mathrm{~km}$ under mirage conditions show a good agreement between experiments and the LWWKD and LWKD models, although the models slightly overestimate the refracted heights of the lower lights. A similar result is now found by EOSTAR, but with a larger overestimate (about $0.5 \mathrm{~m}$ ) for the lowest light. Forand $^{32}$ also presents the maximum intervision ranges (MIVR) for the lights on the ship and concludes that all models (including PIRAM, see below), underestimate the MIVR as compared to the observations. EOSTAR corroborates with this result. Forand finds an excellent agreement between models and experiment when he increases the value of the input parameter air-sea temperature difference (ASTD), which was measured at a single location. This increase is justified by measured ASTD values along the track. When the ASTD is increased, EOSTAR also predicts the observed MIVR values perfectly.

Claverie et al. ${ }^{33}$ focus on three different micrometeorological models, two versions of PIRAM and one version of $\mathrm{L}(\mathrm{W}) \mathrm{WKD}$. The major differences are in the use of exchange coefficients or Charnock's model for the roughness length for stress to initialize the iterative cycle in the models. The Monin-Obukhov lengths calculated with these three models agree within $10 \%$ and within $15 \%$ with EOSTAR. The largest differences are found for the L(W)WKD model. Refraction parameters, such as the MIVR, the mirage interval and the evaporation duct height (EDH) values for radar wavelengths, calculated by all models (including EOSTAR) agree within $10 \%$. Differences are ascribed to the various underlying micrometeorological models rather than the ray tracer module.

Claverie et al. $^{34}$ compare theoretical results of the PIRAM bulk model with experimental results of the MAPTIP campaign. We have first compared the EOSTAR ray tracer to PIRAM. An excellent agreement is found when both models are driven with a profile of constant modified refractivity, which shows that the two ray tracers agree very well. We then calculated MIVR for the various cases as reported by Claverie et al. Our EOSTAR calculations are in good agreement with the PIRAM predictions for the MIVR at different heights, but both models underestimate the observed MIVR values by up to $10 \%$ with EOSTAR having the larger discrepancies. A possible explanation for this underestimate may be that the measurements were made in a coastal area in off-shore wind conditions, which may not be well represented by the bulk models (assuming infinite fetch). As concerns the difference between EOSTAR and PIRAM, it should be noted that the absolute value of the refractivity as calculated by EOSTAR and PIRAM differ by about 1 unit of modified refractivity, which is probably caused by a difference in the temperature profile as calculated by the two models. The difference in absolute value of modified refractivity should not be too influential on the MIVR, since it is the gradient rather than absolute value that controls this phenomenon. However, the different micrometeorological approaches in EOSTAR and PIRAM (e.g., PIRAM uses virtual potential temperature in the iteration loop, whereas EOSTAR uses potential temperature) have apparently caused minor changes in the refractivity gradient resulting in the observed differences.

Dion et al. ${ }^{35}$ publish case studies of the LAPTEX experiment including sensor and target heights. For the two sensor systems given by Dion, the ray tracer of EOSTAR reproduces correctly the maximum geometrical sensor-target ranges of $29.3 \mathrm{~km}$ and $28.8 \mathrm{~km}$ as predicted by IRBLEM under the assumption of a non-wavy surface. If the wave height is taken into account, IRBLEM predicts ranges of 29.0 and $28.6 \mathrm{~km}$, whereas EOSTAR now predicts 28.5 and $28.2 \mathrm{~km}$. For a refractive atmosphere, the comparison between IRBLEM and EOSTAR cannot easily be made, because Dion does not specify the heights of the meteorological sensors, whereas these quantities are required to drive the EOSTAR bulk models. However, indications have been found that EOSTAR and IRBLEM disagree on the classification of subrefractive and super-refractive conditions. Whereas IRBLEM classifies an atmosphere characterized by an ASTD of +2.0 ${ }^{\circ} \mathrm{C}$ as super-refractive, EOSTAR classifies the same atmosphere as sub-refractive due to the very low humidity of $57 \%$ causing a strong humidity gradient close to the surface.

$\operatorname{Stein}^{36}$ presents two case studies of airborne point targets obtained during the LAPTEX experiment. Images recorded in two wavelength bands (3-5 $\mu \mathrm{m}$ and 8-12 $\mu \mathrm{m}$ ) are analyzed and the results are compared to IRBLEM. In the absence of waves, Stein calculates geometric optical sights (GOS) of $44.5 \mathrm{~km}$ and $49.0 \mathrm{~km}$ for the airborne target at two different heights. EOSTAR yields the same values. When the wave heights for the two test cases are entered in EOSTAR, the 
GOS values decrease by 1.1 and $1.5 \mathrm{~km}$, respectively. To obtain the optical sights in a refractive atmosphere, it is assumed that the meteorological parameters were recorded at a standard height of $10 \mathrm{~m}$. However, in that case neither IRBLEM nor EOSTAR predicts the observations. This demonstrates the sensitivity of the refraction effects on the input parameters to the micrometeorological calculations. The two models agree on the prediction of MIVR, but differ on the prediction of the width of the mirage zones. For one test case, EOSTAR gives a better prediction of the mirage zone width than IRBLEM.

Heen and Stark $^{37}$ compare visible and IR (8-12 $\left.\mu \mathrm{m}\right)$ images from ships to ray trajectories obtained with IRBLEM. Three test cases are defined, which all include a complete set of meteorological data obtained from one of the ships. The observed mirage effects are in agreement with the model calculations by IRBLEM, and we also find good agreement using EOSTAR. However, both models find that the lowest visible points of the ship are about $2 \mathrm{~m}$ higher than the actually observed lowest points. In other words, the modeled images predict that a smaller fraction of the ships bow is visible and the bow wave is not visible at all. Another important result of Heen and Stark is that no (measurable) differences where found between the mirages in the visible and IR, which again confirms the conclusion by Dion et al. ${ }^{29}$. The vertical transfer function shown in Figure 17 of the paper is also predicted by EOSTAR, if a wave height of $20 \mathrm{~cm}$ is assumed. The difference between the deepest point in the vertical transfer function for the visible and IR wavelength of a few centimeters is predicted both by IRBLEM and EOSTAR. It is questionable if this small difference can be observed experimentally.

De Jong et al. ${ }^{38}$ present refraction effects for a series of lamps, mounted at different heights between $12 \mathrm{~m}$ and $51 \mathrm{~m}$ above the water surface at a small island Gorgona (Italy), as seen by a CCD camera located at $34 \mathrm{~km}$ distance at the coast of Livorno (Italy). Under non refracting conditions only the lamps above $27 \mathrm{~m}$ could be observed, but during events of strong super refraction, the lowest lights at $12 \mathrm{~m}$ could be observed as well. EOSTAR can successfully predict the occurrence of these super refractive conditions and the associated observation of lights below $27 \mathrm{~m}$. On the other hand, the IRBLEM ray trajectories for the strong super refraction (presented in Figure 30a of De Jong et al.) could not be reproduced using EOSTAR. The latter predicts a smaller maximum detection range of about $41 \mathrm{~km}$ at a height of $50 \mathrm{~m}$, instead of more than $50 \mathrm{~km}$ predicted by IRBLEM. The geometrical horizon and mirage effects presented in Figure 30b can be reproduced by EOSTAR when a wave height of $40 \mathrm{~cm}$ is used. In another experiment at the Livorno site, De Jong et al. use an IR-sensor to image mirage effects of a helicopter at $30 \mathrm{~km}$ range. These effects are well reproduced by EOSTAR.

Heemskerk $^{39}$ uses bulk model 4 in EOSTAR (Smith ${ }^{4}$ with Kondo's ${ }^{40}$ stability functions) to calculate the radar refractivity profiles for the meteorological conditions during the 2004 VAMPIRA project (Germany). These profiles are subsequently used to calculate the one-way propagation factor over a $8.2 \mathrm{~km}$ maritime path for six RF frequencies between 3 and $16 \mathrm{GHz}$ using TERPEM. ${ }^{41}$ A good agreement is found between the measured and calculated transmission, which is an indication of a correct prediction of the refractivity profiles in EOSTAR.

In the above section, we have compared EOSTAR calculations of refractive effects (MIVR, mirage zones, etc) to literature data. The comparisons have been made for several aspects of the calculation, i.e., the underlying micrometeorological models, the refractivity, the refractivity profile, and the ray tracer. As a general conclusion, it can be stated that EOSTAR performs quite well when compared to experimental observations, especially in the prediction of trends, albeit that discrepancies exist with respect to absolute values. The differences between models, and the absolute differences with the observations, mainly result from the micrometeorological modules. Apparently, the prediction of refraction effects is sensitive to small differences in bulk model approach and small errors in the meteorological input parameters to the bulk models.

\section{MODEL PERFORMANCE - OPTICAL TURBULENCE}

As demonstrated in section 2, optical turbulence manifests itself through scintillation. The EOSTAR performance in the prediction of optical turbulence was checked using data from the Validation Measurements for Propagation in the Infrared and Radar (VAMPIRA) trial under the auspices of NATO AC/323 SET-RTG056/RTG32. This trial took place from March 25 to April 5, 2004 near Surendorf, Germany. The SSC 3-4 $\mu \mathrm{m}$ transmissometer was deployed at the site and measured transmission at a rate of $300 \mathrm{~Hz}$ over a $8.2 \mathrm{~km}$ path at a height between 8 and $12 \mathrm{~m}$ above the water surface. The scintillation (values of $C_{n}{ }^{2}$ ) was subsequently calculated using equation (11) (condition $D>>(\lambda R)^{0.5}$ 

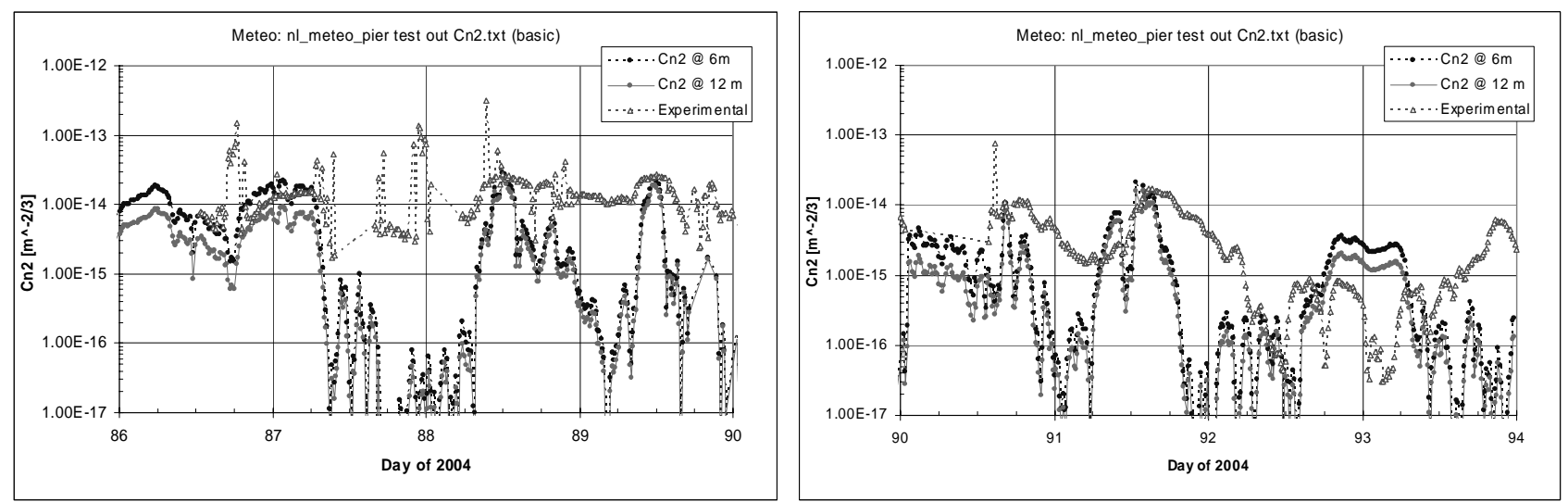

Figure 1: Time series of $C_{n}^{2}$ during the VAMPIRA experiment. The open triangles, connected with dashed lines, refer to the values derived from the transmissometer and are for a height between 8 and $10 \mathrm{~m}$ above the surface. The other two lines represent the calculated values based on the bulk model in EOSTAR at $6 \mathrm{~m}$ and $12 \mathrm{~m}$ above the surface using the meteorological data at the pier.
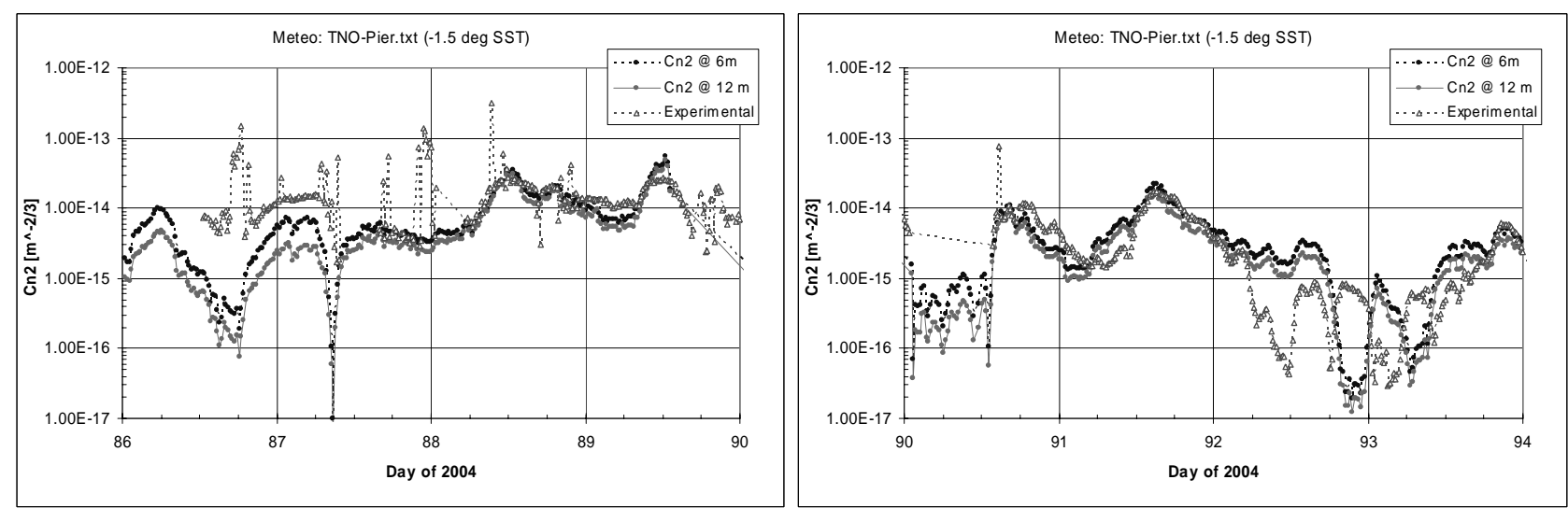

Figure 2: Time series of $C_{n}^{2}$ during the VAMPIRA experiment. The open triangles, connected with dashed lines, refer to the values derived from the transmissometer and are for a height between 8 and $10 \mathrm{~m}$ above the surface (same as Figure 1). The other two lines represent the calculated values based on the bulk model in EOSTAR at $6 \mathrm{~m}$ and $12 \mathrm{~m}$ above the surface, using temperature and humidity from the buoy, wind data from the pier, and applying a correction of $-1.5^{\circ} \mathrm{C}$ to the seawater temperature.
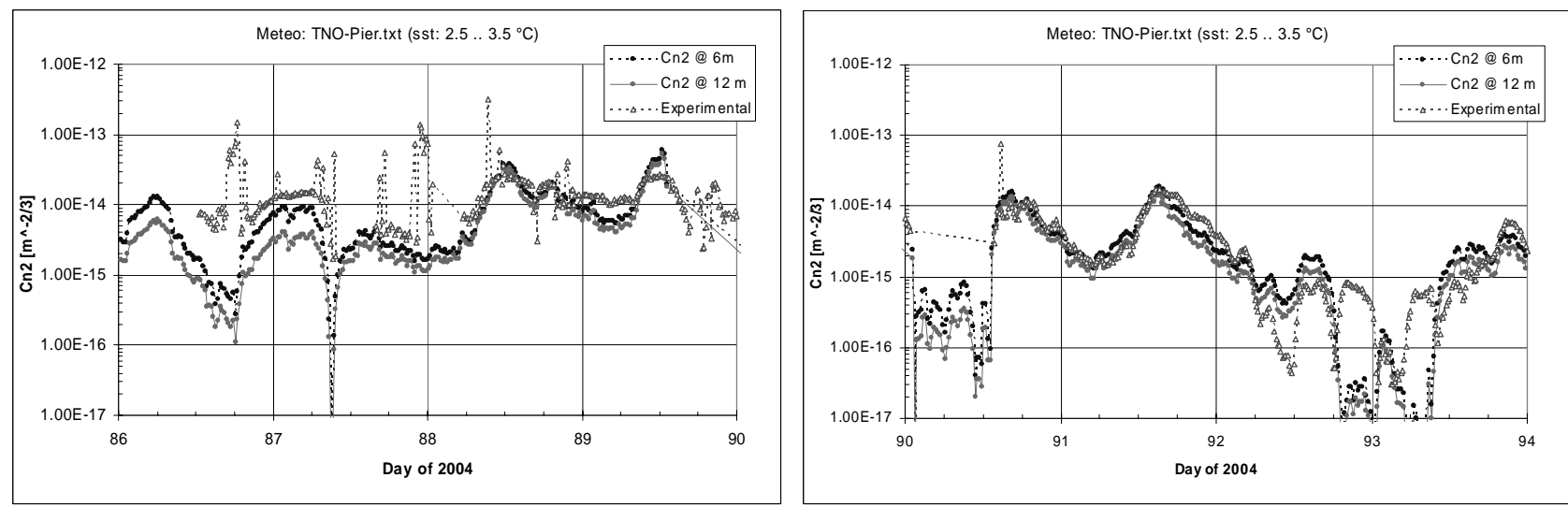

Figure 3: Same as Figure 2 but for a linearly increasing water temperature between $2.5 \mathrm{C}$ and $3.5 \mathrm{C}$. 
fulfilled) and under the assumption of a homogeneous and isotropic atmosphere. The time series of $C_{n}^{2}$ thus obtained will be referred to as 'experimental' data.

The experiment was supported by meteorological sensors at both sides of the propagation path and a buoy in the water half way the path. Sensors at the buoy measured the air temperature and relative humidity at five heights between 0.65 and $5.15 \mathrm{~m}$ above the water level. A thermometer at $1 \mathrm{~m}$ below the water level was used to measure the water temperature. The various meteorological data was fed into the bulk model. EOSTAR is used to calculate time series of $C_{n}{ }^{2}$. These time series will be referred to as 'model' data.

As a first comparison, Figure 1 shows the experimental time series of $C_{n}{ }^{2}$ and two modeled time series. The modeled $C_{n}{ }^{2}$ data is obtained using the meteorological data at one end of the propagation path. For one series, $C_{n}{ }^{2}$ is evaluated at a height of 6 meters, whereas a height of 12 meters is used for the second series. Because $C_{n}^{2}$ degreases monotonically with height the 6 and $12 \mathrm{~m}$ model series should encapsulate the experimental $C_{n}{ }^{2}$ series obtained over a propagation path between 8 and $12 \mathrm{~m}$ height. However, Figure 1 shows that not only the modeled series do not encapsulate the experimental result, but that the trends between model and experiment are also not well reproduced (with the exception of short periods during day 86-87 and day 90).

The calculation of model series of $C_{n}{ }^{2}$ was repeated using the meteorological data from the mid path buoy. Although the agreement with the experiment was marginally better (graphs not shown), neither trends nor absolute values of the experimental $C_{n}{ }^{2}$ were well reproduced by the model calculations. However, this second calculation revealed that there was considerable disagreement (up to $2{ }^{\circ} \mathrm{C}$ ) between the seawater temperature as measured at both sites. When this issue was further explored, we found indications that the seawater temperature has been overestimated. First, the temperature sensor at the buoy is located at 1 meter below the surface. The cold air and the wind may well have caused the surface skin temperature to be lower than $1 \mathrm{~m}$ below the surface. Furthermore, radiometer data was available for part of the experiment showing a surface skin temperature that was systematically lower than measured by the in-situ sensors. Finally, Monin-Obukhov theory applied to the air temperature profile as measured at the buoy, yields surface skin temperatures that are lower than the measured values. For this latter calculation, a logarithmic temperature profile was fitted to the air temperatures measured at the buoy at different levels, with the assumption that the stability correction near the surface vanishes and that the roughness length for temperature is $10^{-3} \mathrm{~m}$ (the effect of using $10^{-4} \mathrm{~m}$ is negligible).

In view of the above reasoning, it was decided to subtract $1.5^{\circ} \mathrm{C}$ of the sweater temperature as measured at the buoy. Furthermore, the air temperature and humidity as measured at $5.15 \mathrm{~m}$ height on the buoy were used, and, because the buoy was not equipped with an anemometer, the wind data as measured at the pier (at one end of the propagation path). The resulting model curves for $C_{n}^{2}$ (for 6 and 12 meters height) are shown in Figure 2. Similar (but slightly worse) results were obtained using a correction of $-2.0^{\circ} \mathrm{C}$ to the seawater temperature. For much of the experiment, Figure 2 shows an excellent match between experimental and model $C_{n}^{2}$, and the experimental curve at times even falls between the two model curves.

The seawater temperature as measured at the buoy varied slowly over the experiment, and increased more or less continuously from approximately 3.5 to $5{ }^{\circ} \mathrm{C}$. As a final calculation, model series of $C_{n}{ }^{2}$ were made using the air temperature and humidity from the buoy, the wind data from the pier, and a surface skin temperature that monotonously increased from 2.5 to $3.5{ }^{\circ} \mathrm{C}$. It is emphasized that these seawater temperatures are purely theoretical and do not correspond to actually measured data. Figure 3 compares the thus obtained model series of $C_{n}{ }^{2}$ to the experimental series. This constitutes our best fit to the experimental series.

The results in figures 2 and 3 suggest that EOSTAR can well reproduce the experimental $C_{n}{ }^{2}$ series, if we accept that the seawater temperature was not measured correctly. However, care must be used when stating this conclusion, since it is always possible that there are other factors (e.g., related to the transmissometer, or the calculation of $C_{n}^{2}$ from the signals) that have resulted in the discrepancy between model and experiment as shown in Figure 1. It should also be noted that a good fit between model and experiment could have been obtained by lowering the air temperature, since it is the ASTD rather than the absolute temperature values that drive the bulk model. However, we did not readily have reason to doubt the experimentalists who have acquired the data, except for the indications presented above that the seawater temperature has been overestimated. As a final remark, we note that even in our best fit, discrepancies remain 
between model and experiment, e.g., during the nights from day 86 to day 87, and from day 92 to day 93 . We have not yet analyzed these periods in more detail.

\section{CONCLUSIONS}

The performance of the EOSTAR model with respect to refraction phenomena and optical turbulence has been assessed. Considering refraction, we have shown on the basis of literature data that our EOSTAR model is comparable with experimental results and comparable with other models. The discrepancies between EOSTAR and other models are attributed to differences in the underlying micrometeorological models. These differences originate from the driving parameter (exchange coefficients or roughness lengths) in the iterative loop, the choice of stability functions, and the choice of fundamental scalars (virtual potential temperature or potential temperature). The discrepancies between EOSTAR (and other models) and experimental data cannot be ascertained beyond doubt. However, we conjecture that errors in the values of the driving meteorological input parameters for the bulk models may play a significant role.

As concerns optical turbulence, the high-frequency variations in the signal of a 3-4 $\mu \mathrm{m}$ transmissometer have been measured over an $8.2 \mathrm{~km}$ path during the VAMPIRA experiment. From these variations, the refractive index structure parameter $C_{n}{ }^{2}$ has been calculated using an equation that accounts for aperture averaging of scintillation. These experimental results are compared with the results predicted by EOSTAR based on the observed meteorological conditions and by assuming a point source and point detector. A good fit between experimental and EOSTAR calculated values of $C_{n}{ }^{2}$ could only be obtained when the seawater temperature was decreased by $1.5{ }^{\circ} \mathrm{C}$ as compared to the measured values. Indications have been provided that justify this change in model input data. The lessons learnt from this exercise are twofold. First, the exercise demonstrates once more that the model results (for refraction and optical turbulence) are quite sensitive to the exact values of the input meteorological parameters. From this follows then that all possible efforts should be made to ensure a proper set of input meteorological parameters. This set includes all relevant properties (i.e., air and sea temperature instead of ASTD only), if possibly measured with redundancy (multiple sensors). Furthermore, it is suggested that all experimental conditions (sensor heights, equipment position, etc.) be recorded carefully.

The sensitivity of the EOSTAR model results (refraction phenomena and optical turbulence) towards the bulk calculations is not unexpected. The bulk model is used to calculate the vertical profile of the atmospheric refractivity, which in turn is the basis to simulate the ray trajectories of the optical path in the refractive atmosphere. These ray trajectories simulate the observation paths of the sensor pixels over tens of kilometers. Due to the long distances involved it is to be expected that the trajectories are sensitive to (small) errors in the bulk models.

The present paper has demonstrated that EOSTAR can reliably predict the occurrence of refraction phenomena and optical turbulence. It is possible to obtain a good agreement between experimental values, based on complex measurements and modeled values obtained from standard meteorological data. $C_{n}{ }^{2}$ is a very important parameter for predicting scintillation, blur and image motion, and thus for predicting the resolution of images that can be obtained with a given sensor for a given meteorological condition.

\section{ACKNOWLEDGEMENTS}

The EOSTAR development is primarily sponsored through various contracts and grants by the Royal Netherlands Navy and the Office of Naval Research. The VAMPIRA trial was organized under the auspices of NATO AC/323 SETRTG056/RTG32.

\section{REFERENCES}

1. Kunz, G.J., M.M. Moerman, A.M.J. van Eijk, S.M. Doss-Hammel and D. Tsintikidis, "EOSTAR: an electro-optical sensor performance model for predicting atmospheric refraction, turbulence, and transmission in the marine surface layer," in Optics in atmospheric propagation and adaptive systems VI, J.D. Gonglewski and K. Stein, Eds., Proc. SPIE Vol. 5237, pp. 81-92, Bellingham, Washington, USA (2003).

2. Kunz G.J., M.A.C. Degache, M.M. Moerman, A.M.J. van Eijk, F.P. Neele, S.M. Doss-Hammel and D. Tsintikidis, "Status and developments in EOSTAR, a model to predict IR sensor performace in the marine environment," in 
Optics in atmospheric propagation and adaptive systems VII, J.D. Gonglewski and K. Stein, Eds., Proc. SPIE Vol. 5572-12, pp. 1-12, Bellingham, Washington, USA (2004).

3. Neele, F.P., Infrared ship signature prediction, model validation and sky radiance, to be published in: Targets and Backgrounds XI: Characterization and Representation, SPIE Proc., Vol. 5811, 2005

4. Smith, S.D., "Coefficients for sea surface wind stress, heat flux, and wind profiles as a function of wind speed and temperature," Journal of Geophysical Research 93(C12), 15,467-15,472 (1988).

5. Liu, W.T., K.B. Katsaros and J.A. Businger, "Bulk parameterization of air-sea exchanges of heat and water vapor including the molecular constraint at the interface," Journal of the Atmospheric Sciences 36(9), 1722-1735 (1979).

6. Högström, U., "Non-dimensional wind and temperature profiles in the atmospheric surface layer: a re-evaluation," Boundary-Layer Meteorology 42, 55-78 (1988).

7. Högström, U., "Review of some basic characteristics of the atmsoheric surface layer," Boundary-Layer Meteorology 78, 215-246 (1996).

8. Fredrickson, P, K.L. Davidson and A.K. Goroch, "Operational bulk evaporation duct model for MORIAH", Naval Postgraduate School, Monterey, CA (2000).

9. Paulson, C.A., "The mathematical representation of wind speed and temperature profiles in the unstable atmospheric surface layer", Journal of Applied Meteorology 9(12), 857-861 (1970)

10. Kunz, G.J., H.J.M. Heemskerk and A.M.J. van Eijk, "Comparison of atmospheric refraction at radar and optical wavelengths", to be published (2005).

11. Beland, R.B., "Propagation through atmospheric turbulence," in The Infrared and Electro-Optical Systems Handbook, Atmospheric Propagation of Radiation, Volume 2, Chapter 2, F.G. Smith, Eds., pp. 157-234, Infrared Information Analysis Center, Ann Arbor, Michagan, USA (1993).

12. Edlén, B., "The dispersion of standard air," Journal of the Optical Society of America 43(5), 339-43 (1953).

13. Hill, R.J. and R.S. Lawrence, "Refractive index of water vapor in infrared windows," Infrared Physics 26(6), 371376 (1986).

14. Davidson, K.L., T.M. Houlihan, C.W. Fairall and G.E. Schacher, "Observation of the temperature structure function parameter, $C_{T}{ }^{2}$, over the ocean," Boundary-Layer Meteorology 15, 507-523 (1978).

15. Fairall, C., G.E. Schacher and K.L. Davidson, "Measurements of the humidity structure function parameters $C_{q}^{2}$ and $C_{t q}$ over the ocean," Boundary-Layer Meteorology 19, 81-92 (1980).

16. Davidson, K.L., G.E. Schacher, C.W. Fairall and A.K. Goroch, "Verification of the bulk method for calculating overwater optical turbulence," Applied Optics 20(17), 498-502 (1981).

17. Kunkel, K.E. and D.L. Walters, "Modeling the diurnal dependence of the optical refractive index structure parameter," Journal of Geophysical Research 88, 10.999-11.004 (1983).

18. Fairall, C.W. and S.E. Larsen, "Inertial-dissipation methods and turbulent fluxes at the air-ocean interface," Boundary-Layer Meteorology 34, 287-301 (1986).

19. Hill, R.J., "Implications of Monin-Obukhov similarity theory for scalar quantities," Journal of the Atmospheric Sciences 46(14), 2236-2244 (1989).

20. Thiermann, V. and A. Kohnle, "Modelling of optically and IR effective atmospheric turbulence," in Atmospheric Propagation in the UV, Visible, IR and MM-Wave Region and Related Systems Aspects, AGARD-CP-454, pp. 19.119.12, Neuilly sur Seine, France (1989).

21. Hill, R.J., G.R. Ochs and J.J. Wilson, "Measuring surface-layer fluxes of heat and momentum using optical scintillation," Boundary-Layer Meteorology 58, 391-408 (1992).

22. Hill, R.J. and G.R. Ochs, "Inner-scale dependence of scintillation variances measured in weak scintillation," Journal of the Optical Society of America 9(8), 1406-1411 (1992).

23. Andreas, E., Eds., Selected papers on turbulence in a refractive medium, SPIE, Bellingham, Washington, USA (1978).

24. Beland, R.R, E.A. Murphy, G.G. Koenig and P.C. Thomas, Comparison of horizontal scintillation measurements and models' in Optical, Infrared, and Millimeter Wave propagation Engineering, SPIE, Belingham, Washington, USA (1988).

25. Ochs, G.R. and T.-I. Wang, "Finite aperture optical scintillometer for profiling with $C_{n}{ }^{2}, "$ Applied Optics 17(23), 3774-3778 (1978).

26. Hill, R.J. and G.R. Ochs, "Fine calibration of large-aperture optical scintillometers and an optical estimate of inner scale of turbulence," Applied Optics 17(22), 3608-3612 (1978).

27. Wang, T.-I., G.R. Ochs and S.F. Clifford, "A saturation-resistant optical scintilometer to measure $C_{n}{ }^{2}$," Journal of the Optical Society of America 68(3), 334-338 (1978). 
28. Hufnagel, R.E., "Propagation through atmospheric turbulence," in The infrared handbook, Capter 6, Wolfe, W.L. and G.J. Zissis, Eds., pp. 6.1-6.56, The Infrared Information and Analysis (IRIA) Center, Environmental Research Institute of Michigan, Ann Arbor, MI, USA (1978).

29. Dion, D., L. Forand, G. Fournier and P. Pace, "Experimental data on near-surface refraction effects at optical wavelengths," in Optics of the air-sea interface: theory and measurements (1749), L. Estep, Eds., SPIE, pp. 120126, Bellingham, Washington, USA (1992).

30. Forand, J.L., D. Dion and J. Bealieu, "MAPTIP: Canada's measurements of refraction effects," in D.H. Höhn, Ed., Symposium on Propagation Assessment in Coastal Environments, AGARD-CP-567, Bremerhafen, Germany, 19-22 September 1994, pp. 24.1-24.6, AGARD (1994).

31. Dion, D., "Refraction effects on EO system detection ranges in coastal environments," in Symposium on Propagation Assessment in Coastal Environments, AGARD-CP-567, Bremerhafen, Germany, 19-22 September 1994, D.H. Höhn, Eds., AGARD, pp. 15.1 - 15.9, Neuille-sur-Seine, France (1994).

32. Forand, J.L., "MAPTIP: refractive effects in the visible and IR," in Image propagation through the atmosphere (2828), Eds., SPIE, pp. 39-49, Bellingham, Washington, USA (1996).

33. Claverie, J., Y. Hurtaud, D. Dion, L. Forand and P. Mestayer, "Influence of meteorological models on refractivity profiles computation in the marine boundary layer," in Propagation electromagnetique dans l'atmosphere du decametricque a l'angstrom, L.B.A. Junchat, Eds., Société des Électriciens et des Électroniciens, pp. 11-16, Paris, France (1997).

34. Claverie, J., B. Tranchant, P. Mestayer, A.M.J.van Eijk and Y. Hurtaud, "Effets de la refraction atmospherique sur la propagation infrarouge dans la basse atmosphere marine comparaison des modeles SeaCluse er PRIAM," in $E$ - $O$ propagation, signature and system performance under adverse meteorological conditions considering out-of-area operations, RTO-MP-1/AC/323/(SET)TP/2, Eds., NATO, pp. 5,1-5,13, Rome (1998).

35. Dion, D., P. Schwering, K. Stein, A. de Jong and H. Winkel, "Low altitude point-target experiment (LAPTEX):analysis of horizon target detection," in EO Propagation, Signature and System Performance under Adverse Meteorological Conditions considering out-of-area operations, AC/323(SET)TP/2, Napels, Italy, 16-19 March 1998, Eds., NATO, pp. 36.1-36.8, Neuille-sur-Seine Cedex, France (1998).

36. Stein, K., "LAPTEX 1996: Analysis of an airborne target over sea," in Europto Conference on Optics in Atmospheric Propagation and adaptive Systems IV (3494), A.D. Devir, A. Kohnle, U. Schreiber and C. Werner, Eds., SPIE, pp. 60-72, Bellingham, Washington, USA (1998).

37. Heen, L.T. and E. Stark, "Examples of refractive effects in visible and infrared", Norwegian Defence Research Establishment, Kjeller (2001).

38. Jong, A.N. de, J. Winkel, M.M. Moerman and R. de Rooy de, "Preliminary report on the TG16 POLLEX trial, May 2001", TNO-FEL Report Nr. FEL-01-A222, September 2001, TNO Physics and Electronics Laboratory, The Hague, The Netherlands (2001).

39. Heemskerk, H.J.M., "RF propagation measurement and model validation," in ECPS 2005, Brest France, $15-18$ March 2005, Brest, France (2005).

40. Kondo, J., "Air-sea bulk transfer coefficients in diabatic conditions”, Boundary-Layer Meteorology 9, 91-112 (1975).

41. Levy, M. F. and K. H Craig, "Millimetre-wave propagation in the evaporation duct", Agard Conference proceedings no. 454, pp. 26-1; 26-10, Copenhagen, Denmark, 9-13 October (1993). 